\title{
A patient with left-sided pleural effusion
}

\section{Case report}

A 56-year-old male patient was admitted to the Akdeniz University Hospital (Antalya, Turkey) with a sudden onset of stabbing epigastric pain, nausea and haematemesis. There was no history of trauma, or systemic or chronic disease. The patient was diagnosed as having gastritis and given appropriate treatment. Routine investigations, such as chest radiography, were not performed at this time.

Two days later, the patient experienced fever, pain on the left side of the thorax, dyspnoea and a worsening of his general condition. Therefore, he was readmitted to the emergency department.

In routine laboratory tests, there were no abnormal findings, with the exception of neutrophilia in peripheral blood. Chest radiography was performed and the results are shown in figure 1.

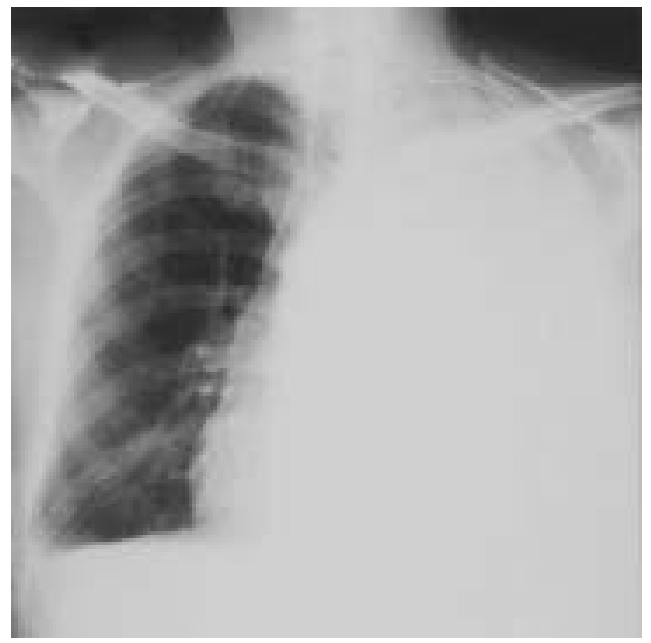

\section{A. Erdogan}

Figure 1

Chest radiograph.

Task 1

Interpret the chest radiograph.
Dept of Thoracic Surgery, Faculty of Medicine, Akdeniz University, Antalya, Turkey.

\section{Correspondence:}

A. Erdogan

Akdeniz Universitesi Hastanesi

GKDC Anabilim Dali

07058 Antalya

Turkey

Fax: 902422274490

E-mail:

erdogana@akdeniz.edu.tr 


\section{Answer 1}

The chest radiograph revealed a left-sided pleural effusion.

Left-sided pleural effusion was also confirmed by ultrasonography. A computed tomography (CT) scan was then performed.

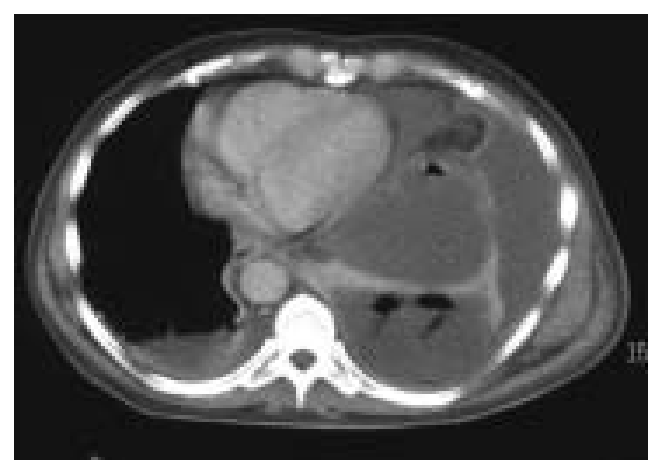

Figure 2

Chest CT scan.

\section{Task 2}

Interpret the CT scan.

\section{Answer 2}

The chest CT scan showed the heart was pushed to the right side and confirmed the presence of left-sided pleural effusion.

Bronchoscopy revealed a narrowing in the basal segment orifices of the left inferior lung lobe. Upper abdominal ultrasonography was reported as normal. During gastroscopy, the wall and mucosa of the gastric fundus were observed to be normal, but the wall and mucosa of the gastric corpus were oedematous and erosive sites were present. The results of an oesophagoscopy and duodenoscopy were found to be normal.

Thoracentesis suggested a haemothorax and $\sim 500 \mathrm{~mL}$ of haemorrhagic pleural effusions was drained via a thoracostomy tube. In spite of this drainage, the patient deteriorated. No expansion of the lung was observed on chest radiography. Subsequently, the patient was referred for consultation at the Dept of Thoracic Surgery (Akdeniz University Hospital).

\section{Task 3}

Based on the results presented so far, suggest a diagnosis and the next investigative procedure. 


\section{Answer 3}

A diagnosis of empyema was made and a thoracotomy was planned.

During the thoracotomy, pleuritis with fibrin deposition was observed. Parts of the omentum and gastric fundus were herniated into the thoracic region from the Bochdalek foramen, and the gastric fundus was perforated (figure 3).
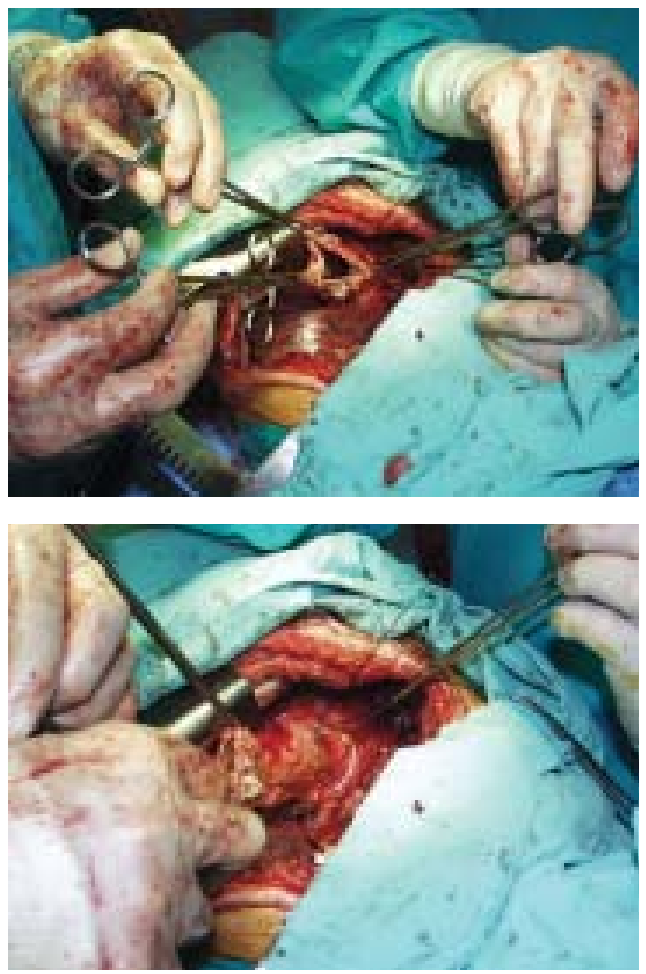

Figure 3

Bochdalek herniation of the stomach and perforated fundus observed during thoracotomy.

The perforated areas in the gastric fundus and diaphragm were repaired successfully using surgical sutures.

Pleuritis developed post-operatively, and was treated successfully with cefazolin ( 3 gday $\left.^{-1}\right)$, ami$\operatorname{kacin}\left(1\right.$ gday $^{1}$ ) and metronidazole (2 gday ${ }^{1}$ ) for 7 days. A culture of pleural effusion revealed mixed bacterial flora.

No further complications were encountered during hospitalisation of the patient. The patient was discharged from hospital at the end of the 12th day. The patient was in good health at the 1-, 3- and 6-month follow-ups.

\section{Discussion}

Congenital diaphragmatic hernia appears on the left side, through the foramen of Bochdalek, usually in newborns and during early infancy. It is characterised by tachypnoea, cyanosis and respiratory failure [1]. Adult left-sided diaphragmatic hernia is rarely encountered, but, when observed, it often results from preceding trauma $[1,2]$.

The patient presented here is a very rare case of a left-sided diaphragmatic hernia that developed through the Bochdalek foramen. The patient's stomach and omentum were herniated into the thorax, resulting in the development of pleural effusion and empyema due to a perforated stomach fundus.

Delayed Bochdalek hernia usually occurs as a result of right-sided defects of the diaphragm. The positioning of the liver contributes partly to the closure of diaphragmatic defect. Although delayed herniations of the left side have been reported previously, there have been no cases occurring after puberty, and especially in those who are middle-aged and elderly [3]. Patients with delayed Bochdalek hernia usually experience recurrent pulmonary infections, dyspnoea, wheezing, chest and abdominal pain, loss of appetite, nausea, vomiting, diarrhoea and general deterioration [4-6]. Gastric and intestinal volvulus, strangulation of the gastric fundus, acute gastric dilatation and secondary tension pneumothorax have also been reported [7-9].

Incidents such as episodic sets and blunt trauma, which may produce extreme pressure changes, may precipitate the passage of the stomach through the diaphragmatic defect into the thoracic cavity.

In the current case, the patient had a previous history of common cold and intensive coughing, but there was no blunt trauma to the abdomen or thorax. He was not treated appropriately after his first admission due to an incorrect diagnosis of peptic ulcer, resulting from symptoms of epigastric pain and signs compatible with peptic ulcer. Hence, he was discharged home without undergoing barium study or chest radiography. In such cases, a chest radiograph could be life saving, as it may lead to the proper diagnosis.

Gastroscopy revealed oedema of the mucosa, but no perforation. Pleural effusion and minimal pericardial effusion were observed using thorax CT scanning, and there were no signs of pneumothorax. As a result, a diagnosis of gastric perforation was excluded. The patient was assumed to have haemothorax, and a thoracostomy tube was inserted into thorax. However, this was a misleading procedure.

In conclusion, patients presenting with similar symptoms should undergo a detailed examination, and chest radiography must be performed even in asymptomatic patients. 


\section{References}

1. Quah BS, Hashim I, Simpson H. Bochdalek diaphragmatic hernia presenting with acute gastric dilatation. J Pediatr Surg 1999; 34: 512-514.

2. Brown GL, Richardson JD. Traumatic diaphragmatic hernia: a continuing challenge. Ann Thorac Surg 1985; 39: 170-173.

3. Campbell DN, Lilly JR. The clinical spectrum of right Bochdalek's hernia. Arch Surg 1982; 117: 341-344.

4. Radin DR, Ray MJ, Halls JM. Strangulated diaphragmatic hernia with pneumothorax due to colopleural fistula. AJR Am J Roentgenol 1986; 146: 321-322.

5. Payne JH Jr, Yellin AE. Traumatic diaphragmatic hernia. Arch Surg 1982; 117: 18-24.

6. Kirshner SG, Burko HO, Neill JA, Stahlman M. Delayed radiographic presentation of congenital right diaphragmatic hernia. Radiology 1975; 115: 155-156.

7. Richard JS, Jeffrey GJ. Tension pneumothorax secondary to a gastropleural fistula in a traumatic diaphragmatic hernia. Chest 1991; 99: 247-249.

8. Karanikas ID, Dendrinos SS, Liakakos TD, Koufopoulos IP. Complications of congenital posterolateral diaphragmatic hernia in the adult. Report of two cases and literature review. J Cardiovasc Surg 1994; 35: 555-558.

9. Leitao B, Mota CR, Enes C, Ferreira P, Vieira P, Requeijo D. Acute gastric volvulus and congenital posterolateral diaphragmatic hernia. Eur J Pediatr Surg 1997; 7: 106-108. 